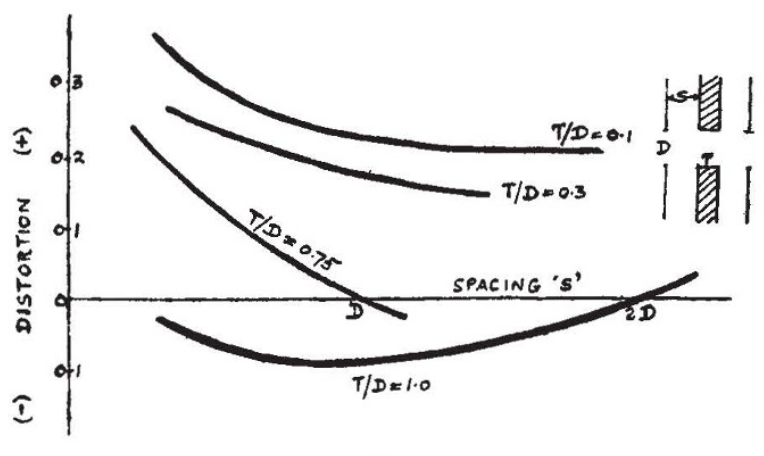

Fig. 2

increasing strength, there is a return to the original type of distortion, indicating that the electrons cross the axis twice on their way to the screen.

An effect of a similar kind occurs with a magnetic projection lens and can be readily demonstrated. The 'pincushion' pattern becomes less distorted with increasing lens-strength, and for one particular value of lens-power no distortion is present. With further increase in strength the magnification decreases and 'barrel' distortion appears; this later gives place to bad 'pincushion' effects with increasing rise in lens power.

It is interesting to note that the effect produced by introducing the thick middle electrode is the same as that shown by Hillier ${ }^{4}$ to hold for a combination of two lenses of approximating equal focal length separated by a distance equal to their focal length.

The screening effect of the thick central electrode appears to produce a lens on either side of it, which, in combination, eliminate the distortion.

It is hoped to publish these results in full at a later date.

Electrotechnics Laboratory,

T. Mulvey

University of Manchester.

George Holt Physics Laboratories,

L. $\mathrm{J}_{\mathrm{ACOB}}$

University of Liverpool.

'Physic8, 7, 451 (1936).

${ }^{2} Z$. Instr., 59, 71 (1939).

${ }^{3}$ Proc. Phys. Soc. (in the press).

๑J. Appl. Phys., 17, 411 (1946).

\section{Stopping Power of Liquid Water for $\alpha$-Particles}

THE mean stopping power of liquid water averaged over the whole range has been determined for natural $\alpha$-particles by Michl $^{1}$ and by Philipp ${ }^{2}$. These authors find values for the molecular stopping power which are higher by about 20 and 14 per cent respectively than those predicted by the Bragg law and the accepted atomic stopping powers of hydrogen and oxygen. Philipp (loc. cit.) has also shown that the stopping power of water vapour accords with the Bragg law. The tendency among physicists has, however, been to treat the Bragg law as a more reliable guide to the stopping power of liquid water than the experiments.

Water occupies a fundamental position in radiobiology as the most important constituent of all living cells. Any uncertainty as to its stopping power is, in consequence, most undesirable, especially from the point of view of practical dosimetry and radiotherapeutics. Experiments have therefore been carried out in this laboratory with a view to the redetermination of stopping power. The method used differs in principle from both the photographic tech. nique of Michl and the microscopic technique of Philipp. It has the advantage that the necessity of locating the surface of a layer of water to within a very few microns is eliminated.

An aqueous solution of polonium in $0.5-1.5 \mathrm{~N}$ hydrochloric acid acts as a thick source of $\alpha$-particles which are detected by a thin-window Geiger counter. The variation of the effective thickness of the source with the distance in air from the surface of the liquid to the counter may then be used to measure the ratio of the stopping powers of the liquid and the laboratory air. To determine the effective thickness of the source, it is only necessary to know the counting. rate, the area of liquid surface exposed to the counter and the strength of the solution. The latter quantity is measured by evaporation of a known mass of solution to dryness. The counting-rate due to the dried source is then compared with that due to the solution under similar geometrical conditions.

Preliminary results indicate that for 4-5 $\mathrm{MeV}$. $\alpha$-particles the instantaneous stopping power per molecule of liquid water relative to that of air per atom is $1 \cdot 71$. The experimental error of the determination is estimated to be less than \pm 5 per cent. This value is approximately 13 per cent higher than the value, $1 \cdot 51$, predicted by the Bragg law. On the basis of the Bethe-Bloch theory, it agrees within experimental error with the mean stopping powers obtained by Michl and by Philipp. This suggests that the higher value of the stopping power of water should be used in the assessment of the radiotherapeutic effects of heavy particles.

A detailed description of these experiments will be published elsewhere.

The assistance of a grant from the Medical Research Council is gratefully acknowledged.

$$
\begin{aligned}
& \text { R. K. APPLEYARD } \\
& \text { Department of Radiotherapeutics, } \\
& \text { University of Cambridge. } \\
& \text { Oct. 1. } \\
& \text { 'Michl, W., Sitz. K. Akad. Wiss. Wien, } 123 \text { (IIA), } 1965 \text { (1914). }
\end{aligned}
$$

'Philipp, K., Z. Phys., 1'7, 23 (1923).

\section{Methylene Bromide as a Quenching Agent in Geiger-Müller Counters}

GEIGER-MüLMER counters filled with methylene bromide and argon are $\mathrm{known}^{1}$ to be self-quenching and to possess long sloping plateaux. Measurements have now been made of the $\beta$-counting-rate as a collimated source was moved diametrically across the mica end-window of a $\beta$-ray counter so that $\beta$-particles entered the counter parallel to the centre wire. Two counters $A$ and $B$ of identical construction were used for these measurements, $A$ containing argon at a pressure of $97 \mathrm{~mm}$. of mercury and ethyl alcohol at a pressure of $9.7 \mathrm{~mm}$. of mercury, and $B$ containing argon at a pressure of $74 \mathrm{~mm}$. of mercury and methylene bromide at a pressure of $7.4 \mathrm{~mm}$. of mercury. It was found that the efficiency of counter $B$ was greatest for particles entering the counter near the centre wire, but even then was less than that of counter $A$ by a factor of about three.

The usual method ${ }^{2}$ for calculating the theoretical efficiency of $\beta$-ray counters assumes that, in order to cause a count, only one electron need be produced in the counter by a $\beta$-particle. According to this 